Document downloaded from:

http://hdl.handle.net/10251/121744

This paper must be cited as:

Larrea-Luzuriaga, RA.; Gutierrez Campo, AM.; Sanchis Kilders, P. (2018). Design method for high performance grating couplers in photonic integrated circuits. Optical and Quantum Electronics. 50(9):1-9. https://doi.org/10.1007/s11082-018-1596-8

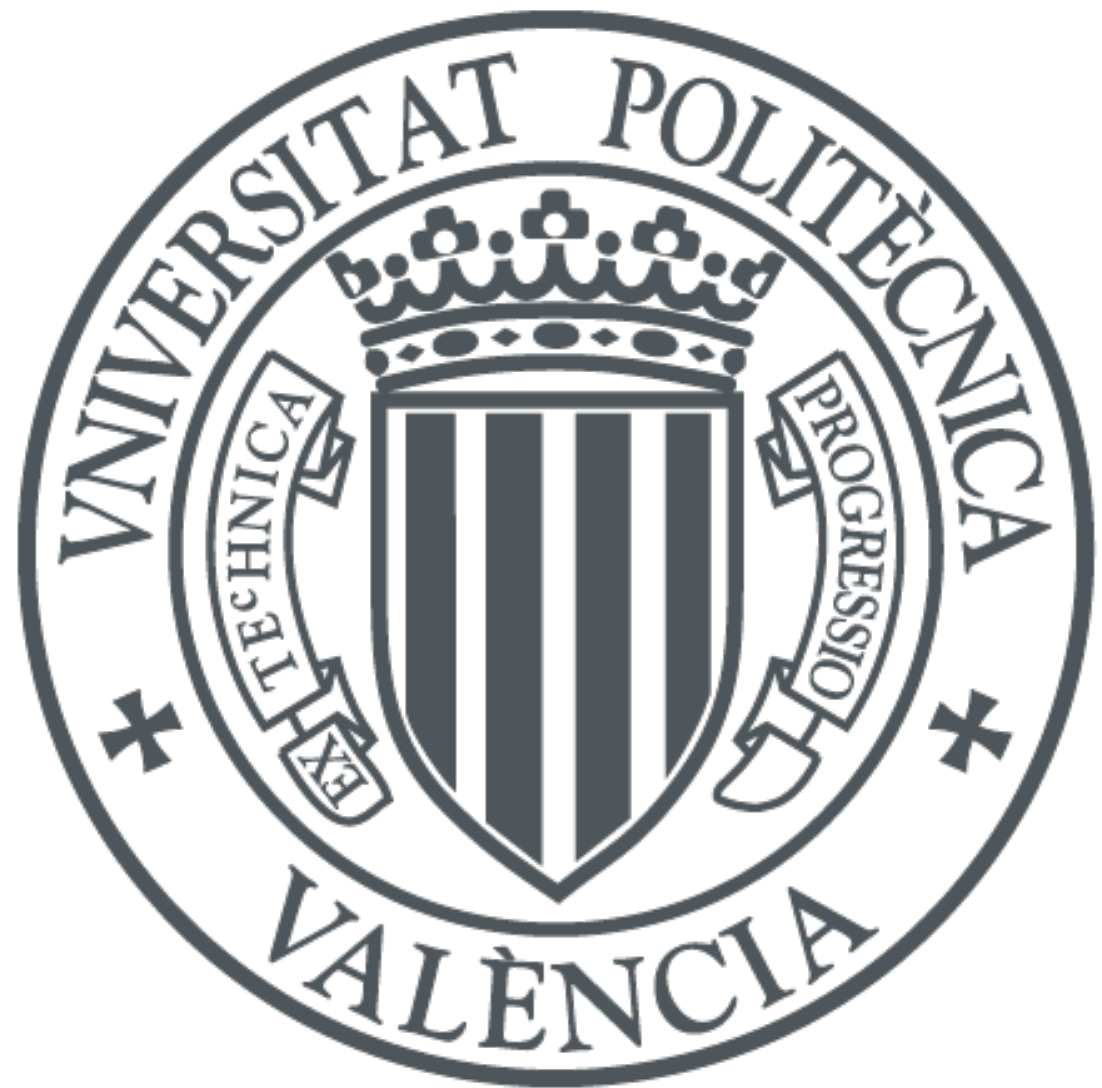

The final publication is available at

https://doi.org/10.1007/s11082-018-1596-8

Copyright Springer-Verlag

Additional Information 


\title{
Design method for high performance grating couplers in photonic integrated circuits
}

\author{
Roberto Larrea, Ana Maria Gutierrez and Pablo Sanchis* \\ Nanophotonics Technology Center, Universitat Politècnica de València, Valencia 46022, \\ Spain \\ *Email:pabsanki@ntc.upv.es
}

\begin{abstract}
The optimization of grating couplers is usually realized by multiple simulations using specific computational software for this task. Many grating parameters must be analyzed and designed to get the maximum coupling efficiency and the transmission spectrum centred at the wavelength of operation. However, these simulations may take a long time and consume high computational resources depending on the simulation resolution. This work is focused on finding a method to optimize the grating parameters with the lowest number of simulations. In this way, closed-form expressions are presented to get the optimal values for the period and fill-factor, which are the main parameters in the grating design. The usefulness of the proposed approach is shown for the design of silicon grating couplers operating at $1.31 \mu \mathrm{m}$ and $1.55 \mu \mathrm{m}$ and both TE and TM polarizations.
\end{abstract}

Keywords: grating coupler, photonic integrated circuits, silicon photonics

\section{Introduction}

Silicon photonics is a technology that has experienced a great development in the last years due its compatibility with the CMOS fabrication process and the optical properties of the material. The compatibility with the microelectronic technology allows a mass fabrication process with a low cost using a material abundant in the nature as silicon [1]. Moreover, the high index contrast between the silicon and silica allows the confinement of light in optical waveguides with submicron sizes and the design of complex structures for diverse applications. However, most of these structures are quite sensitive to variations of their geometry [2].

The submicron size of optical waveguides makes challenging the coupling of light with other components of larger size such as the optical fiber. Therefore, several coupling mechanisms have been investigated for an efficient coupling. One of the most attractive mechanisms is the use of grating couplers for coupling light out of plane [3]. Many studies have been made about grating couplers, all of them focused on achieving the maximum coupling efficiency. Standard silicon grating couplers have been traditionally designed through an intensive optimization by simulations [4-7]. More recently, new methods of optimization have been shown to reduce the number of simulations and improve the coupling efficiency as gradient-based methods [8], optimization strategies based on simultaneous apodization of the principal design parameters [9], or inverse design technics where the user can define initially a set of performance metrics [10]. In all cases, some parameters of the grating coupler structure have been considered in the design and several simulations have been performed to maximize the coupling efficiency. 
This paper proposes a method to calculate in a semi-analytical way the optimal values for the most important parameters of the grating coupler structure. To achieve this goal, the influence of the effective index of the propagating mode in the grating coupler on the optimum period has been analyzed and closed-form expressions have been formulated to get both the period and the fill-factor. Therefore, the optimal value for the effective index is obtained which ensures the maximum coupling efficiency and the spectrum centred at the operational wavelength.

The method has been validated with some designs of silicon-on-insulator (SOI) grating couplers. High coupling efficiencies have been achieved with values comparable to the ones obtained through extensive 2D-FDTD simulations. Therefore, a coupling efficiency of $57 \%$ at $1.55 \mu \mathrm{m}$ and $59 \%$ at $1.31 \mu \mathrm{m}$ has been achieved for TE polarization. Moreover, it has been shown that the optimum parameters for TM polarization may also be estimated from the parameters obtained for TE polarization. In such a way, a coupling efficiency of $50 \%$ at $1.55 \mu \mathrm{m}$ and $60 \%$ at $1.31 \mu \mathrm{m}$ has been achieved for TM polarization.

\section{Grating coupler performance}

Grating couplers are periodic structures that change their configuration or geometry usually with a variation of the refractive index or a surface corrugation. This perturbation affects to the phase of a wave impinging on the grating coupler so that it will acquire a periodic behaviour [11]. The resulting wave can be expanded into a Fourier series, where each harmonic is propagating at a different spatial direction and represents a diffracted wave. The number of allowed diffracted waves is determined by the Bragg condition [12]:

$$
\vec{k}_{m}=\vec{k}_{\text {inc }}+m \vec{K}
$$

where $\vec{k}_{m}$ is the wavevector of the diffracted wave, $\vec{k}_{i n c}$ is the wavevector of the incident wave and $\vec{K}$ is the grating vector, which is determined by the period, $\Lambda$, of the grating. The integer $m$ describes the diffracted order $(m=0, \pm 1, \pm 2 \ldots \pm \infty)$ as it can be observed in Fig. 1 .

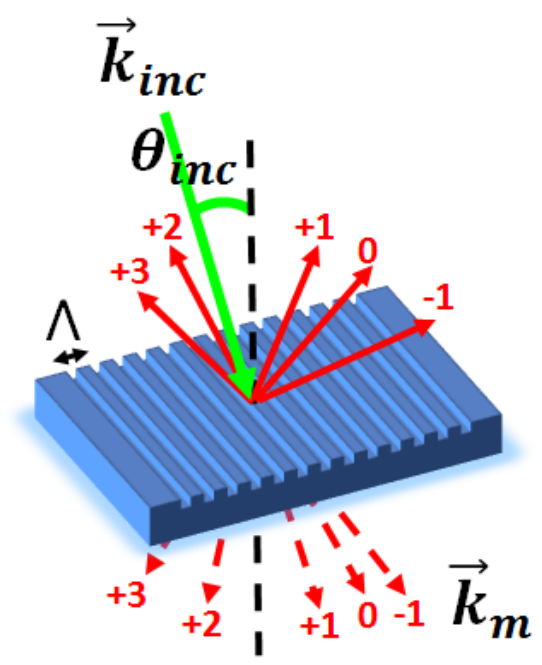

Figure 1.- Incident wave on a surface with a periodic structure and resulting diffracted waves.

Grating couplers are designed for efficient coupling to a photonic waveguide from an optical fiber. Due to the reciprocity theorem, the same results can be considered for coupling from the waveguide to the fiber. The grating coupler is a three dimensional (3D) structure (Fig. 2(a)). However, a study in a bidimensional (2D) plane can be made (Fig. 2(b)) because the waveguide width is much longer than the effective wavelength, so a waveguide with an infinite width can be considered [13]. The first-order of diffraction $(m=1)$ is used to excite the propagating mode of the grating coupler characterized by an effective index of $n_{\text {effg. }}$. Furthermore, the incident wave from the fiber is slightly detuned by a certain 
angle, $\theta_{\text {inc }}$, from the vertical direction to avoid the second-order diffraction, as it can be seen in Fig. 2(b). Thereby, the Bragg condition can be reduced to:

$$
n_{\text {effg }} \frac{2 \pi}{\lambda}=n_{\operatorname{SiO} 2} \frac{2 \pi}{\lambda} \sin \theta_{i n c}+\frac{2 \pi}{\Lambda}
$$

from which the grating period or pitch for a specific wavelength, $\lambda$, is easily derived as

$$
\Lambda=\frac{\lambda}{n_{e f f g^{-}} n_{S i O 2} \sin \theta_{i n c}}
$$

where $n_{s i o 2}$ is the refractive index of the silica deposited above the grating coupler.
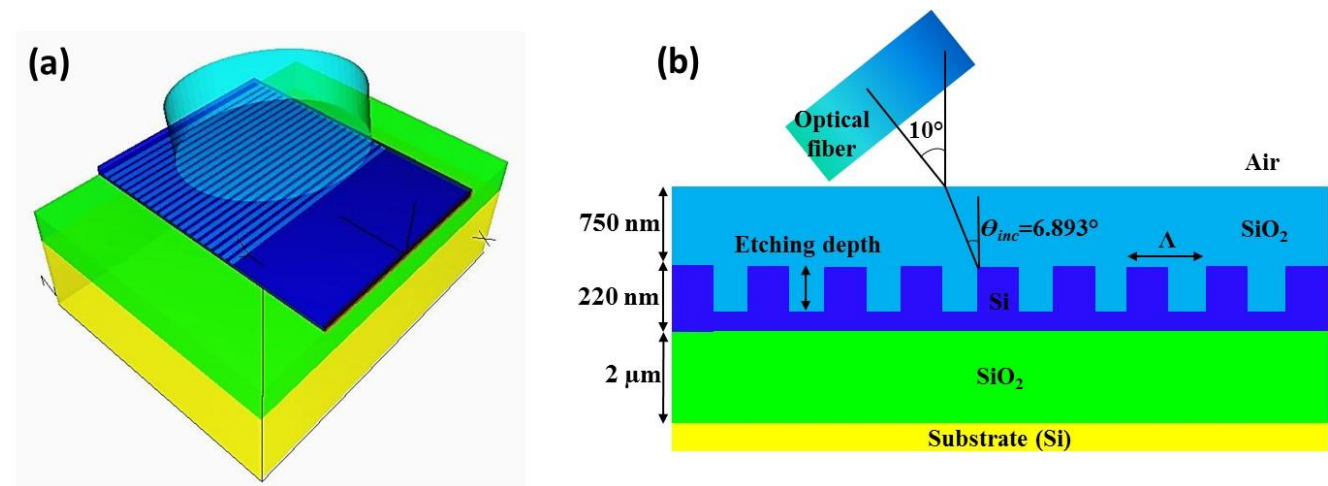

Figure. 2 (a) Grating coupler 3D view and (b) cross section with design parameters.

The effective index of the propagating mode in the grating coupler is the key design parameter to find the optimum grating period by using Eq. (3). However, this parameter cannot be directly calculated and will depend on the grating coupler structure. The usual approach is to calculate the effective index of both waveguides that form the grating in its etched, $n_{\text {effep }}$, and non-etched, $n_{\text {effnep }}$, parts, as shown in Fig. 3, so that the effective index of the propagating mode as a function of fill-factor $F f$ is obtained by:

$$
n_{e f f g}=n_{e f f n e p} \cdot F f+n_{e f f e p} \cdot(1-F f)
$$

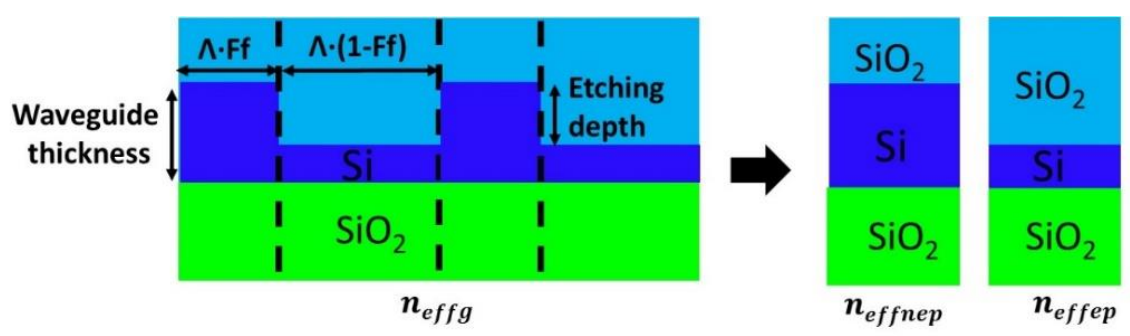

Figure. 3. Decomposition of the grating coupler structure in two planar waveguides to calculate the effective index from the etched and non-etched parts.

However, the coupling efficiency cannot be obtained from the Bragg equation defined by Eq. (2) [14]. For an efficient coupling, several parameters must be considered in the design. This is the case of the fill-factor, the etching depth of the grating and the waveguide thickness, all of them illustrated in Fig. 3. On one hand, the fill-factor determines a proportional relation between the etched and non-etched parts. On the other hand, the etching depth establishes the perturbation grade of the light in the grating while the waveguide thickness affects to the effective index of the medium in which the light propagates. Therefore, all these parameters will have an influence on the effective index of the propagating mode in the grating. 


\section{Proposed design method}

The proposed method is based on an alternative approach to find the optimum effective index of the propagating mode in the grating coupler structure. Therefore, the influence of the effective index has been firstly analyzed in the grating structure shown in Fig 2(b) by assuming an etching depth of $70 \mathrm{~nm}$ and a fill-factor of 50\%. First, the optimum period has been achieved for a wavelength range between $1.26 \mu \mathrm{m}$ and $1.60 \mu \mathrm{m}$ and TE polarization. The optimum period, shown in Fig. 4(a), has been obtained by means of 2D-FDTD simulations for having maximum coupling efficiency and the optical spectrum centred at the target wavelength. With the optimized values of the period for each wavelength, the optimum effective index of the propagating mode in the grating can be derived from Eq. (3). Figure 4(b) shows the obtained optimum effective index values as a function of wavelength. The optimum values are compared in Fig. 4(b) with the effective index calculated by using Eq. (4).
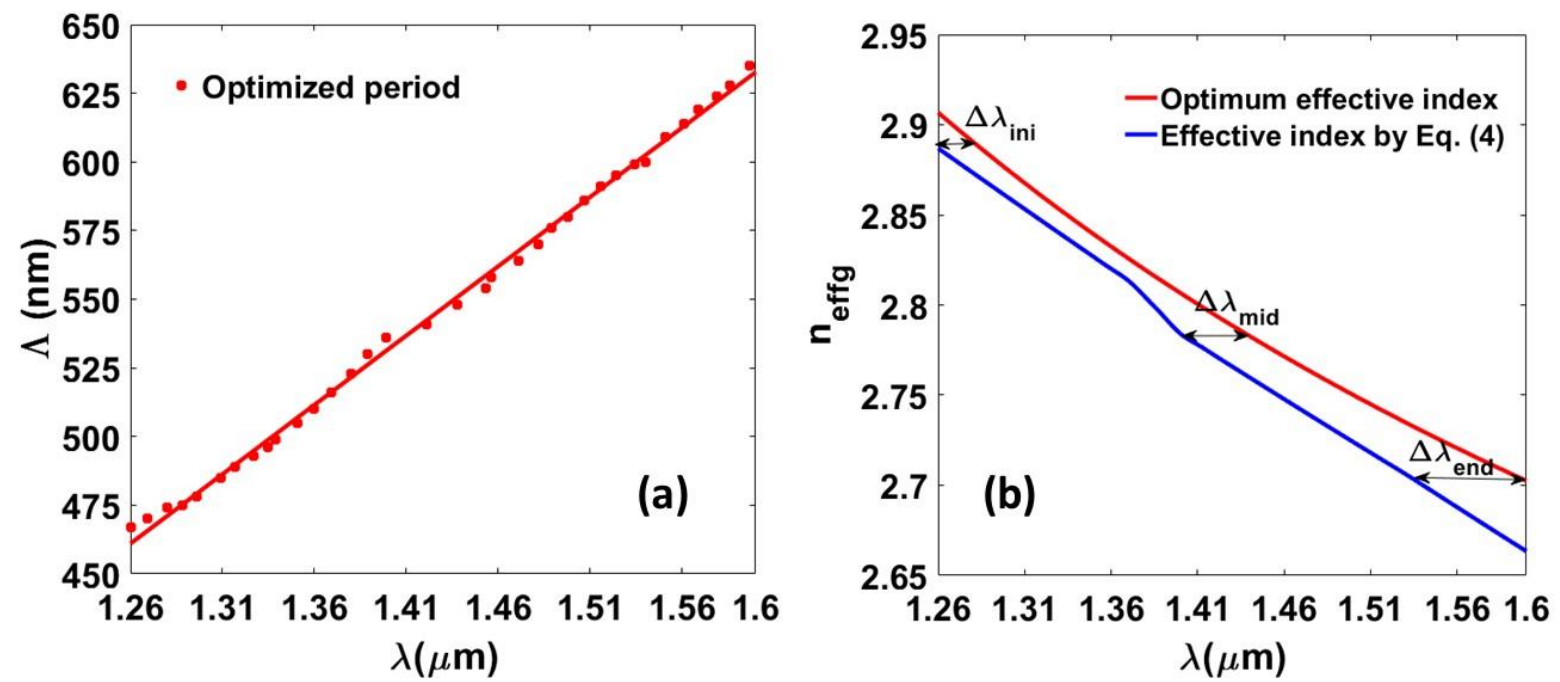

Figure. 4 (a) Optimized period and (b) optimum effective index extracted from the optimized period and effective index calculated by Eq. (4) as a function of wavelength.

From the results shown in Fig. 4(b), it can be observed that the same value of effective index is achieved for different wavelengths. Therefore, the optimum effective index at the target wavelength of operation can be obtained by considering a shifted wavelength:

$$
\lambda^{\prime}=\lambda-\overline{\Delta \lambda}_{T E}
$$

where the factor $\overline{\Delta \lambda}_{T E}$ can be approximated by an average value in the wavelength range of interest. The initial, medium and final wavelengths are considered, as depicted in Fig. 4(b):

$$
\overline{\Delta \lambda}_{T E}=\frac{\Delta \lambda_{\text {ini }}+\Delta \lambda_{\text {med }}+\Delta \lambda_{\text {end }}}{3}
$$

which gives a value of $\overline{\Delta \lambda}_{T E}=36 \mathrm{~nm}$ for the considered grating coupler structure. Such average value will allow the calculation of the optimum effective index in the whole wavelength range between 1.26 $\mu \mathrm{m}$ and $1.60 \mu \mathrm{m}$. Therefore, the design process will be very fast and efficient. The design process steps will be:

1) Define the desired wavelength to have maximum coupling efficiency and the transmission response of the grating centred around that wavelength, for instance, $\lambda=1310 \mathrm{~nm}$.

2) Simulate or calculate the effective indexes of the planar waveguides corresponding to the etched and non-etched parts of the grating, as depicted in Fig. 3, at the shifted wavelength defined by 
Eq. (5), i.e. $n_{\text {effep }}\left(\lambda-\overline{\Delta \lambda}_{T E}\right)$ and $n_{\text {effnep }}\left(\lambda-\overline{\Delta \lambda}_{T E}\right)$. In the example, $n_{\text {effep }}\left(\lambda^{\prime}=\right.$ $1274 \mathrm{~nm})$ and $n_{\text {effnep }}\left(\lambda^{\prime}=1274 \mathrm{~nm}\right)$.

3) The optimum effective index of the propagating mode in the grating coupler structure will then be obtained with:

$$
n_{\text {effg-opt }}=\frac{n_{\text {effep }}\left(\lambda-\overline{\Delta \lambda}_{T E}\right)+n_{e f f n e p}\left(\lambda-\overline{\Delta \lambda}_{T E}\right)}{2}
$$

In the example, $n_{\text {effg-opt }}=2.8774$.

4) With the optimum effective index, $n_{\text {effg-opt }}$, the optimum period will be calculated from the Bragg equation:

$$
\Lambda_{o p t}=\frac{\lambda}{n_{e f f g-o p t}-n_{S i O 2} \sin \theta_{i n c}}
$$

In the example, $\theta_{\text {inc }}=6.893^{\circ}, n_{S i O 2}=1.446$ and so $\Lambda_{\text {opt }}=485 \mathrm{~nm}$.

5) Finally, the optimum filling factor is calculated by:

$$
F f_{o p t}=\frac{n_{\text {effg-opt }}-n_{\text {effep }}(\lambda)}{n_{\text {effnep }}(\lambda)-n_{\text {effep }}(\lambda)}
$$

In the example, we need to calculate first $n_{\text {effep }}(\lambda=1310 \mathrm{~nm})$ and $n_{\text {effnep }}(\lambda=1310 \mathrm{~nm})$ which gives then $F f_{\text {opt }}=58.5 \%$.

In summary, once the factor $\overline{\Delta \lambda}_{T E}$ has been determined for a certain wavelength range, the optimum period and filling-factor can be calculated in a fast and efficient way for any wavelength following the procedure previously described. The obtained values will ensure a coupling efficiency close to the maximum and an optical bandwidth centred around the target wavelength. Furthermore, a relationship between TE and TM polarizations can also be found by comparing the variation of the optimum period with wavelength for both polarizations, which is given by

$$
\frac{\partial \Lambda_{T M}}{\partial \lambda} \approx 2 \frac{\partial \Lambda_{T E}}{\partial \lambda}
$$

for the considered grating coupler. According to Eq. (10), the variation of the period with wavelength for TM polarization is two times higher than for TE polarization so that it can deduced that the factor used to obtain the shifted wavelength in Eq. (5) will also be affected by a factor of two:

$$
\overline{\Delta \lambda}_{T M}=2 \overline{\Delta \lambda}_{T E}
$$

which gives a value of $\overline{\Delta \lambda}_{T M}=72 \mathrm{~nm}$. 

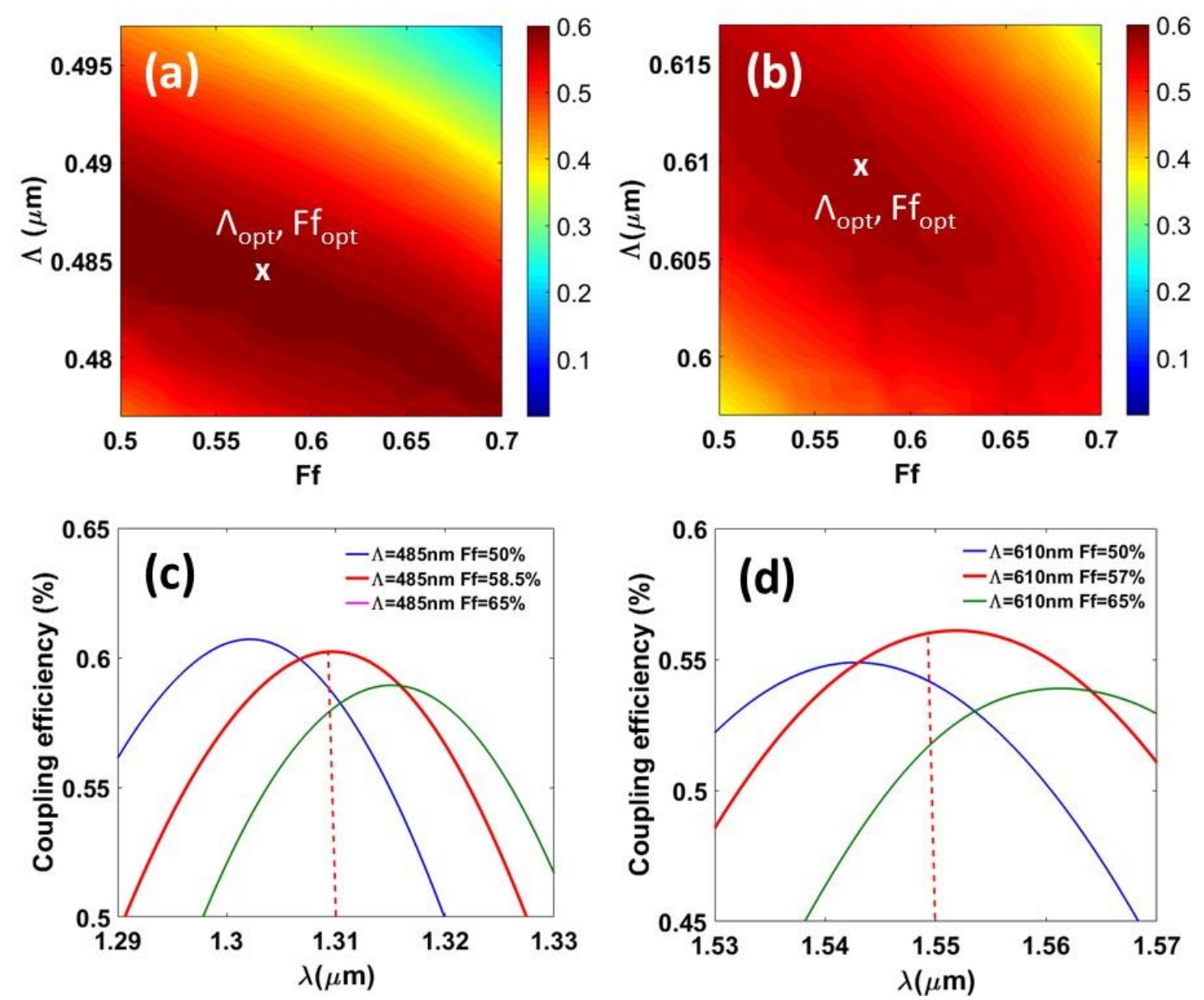

Figure. 6. Simulated coupling efficiency for TE polarization as function of the period and fill-factor for (a) $1310 \mathrm{~nm}$ and (b) $1550 \mathrm{~nm}$. The optimal values $\left(\Lambda_{\text {opt }}, F f_{\text {opt }}\right)$ obtained with the proposed design method are also shown. Transmission spectrum of the grating coupler for (c) $1310 \mathrm{~nm}$ and (d) $1550 \mathrm{~nm}$ for the optimum period and different fill-factor values.

\section{Results and comparison with simulations}

The performance of the grating coupler shown in Fig. 2(b) and designed with the proposed method for operation at a wavelength of $\lambda=1310 \mathrm{~nm}$ has been compared with simulations. Figure 6(a) shows a contour map of the coupling efficiency as function of period and fill-factor obtained by means of 2DFDTD simulations. The optimal values $\left(\Lambda_{o p t}=485 \mathrm{~nm}, F f_{o p t}=58.5 \%\right)$ derived with the proposed design method are also shown and give rise to a coupling efficiency of $59 \%$. It can be seen that the obtained values are very close to the maximum coupling efficiency derived from simulations. The design method has also been applied for a wavelength of $\lambda=1550 \mathrm{~nm}$. In this case, the effective index of the propagating mode in the grating coupler is $n_{\text {effg-opt }}=2.7157$ which yields to an optimum period of $\Lambda_{o p t}=610 \mathrm{~nm}$ and a fill-factor of $F f_{o p t}=57 \%$. Figure 6(b) shows the comparison with the results obtained by simulations and confirm that the designed parameters are very close to the maximum coupling efficiency. The obtained coupling efficiency is in this case $57 \%$. It should be noticed that in the simulation results shown in Fig. 6(a) and Fig. 6(b), the coupling efficiency has been simulated without taking into account if the transmission spectrum of the grating is really centred at the target wavelength. Figure 6(c) and Fig. 6(d) shows the transmission spectrum of the grating for the optimum period obtained by the proposed method and for different values of the filling factor. It can be seen that the filling factor is critical in the position of the spectrum and that the optimum value obtained by the proposed method ensures that the spectrum is centred around the target wavelength. 

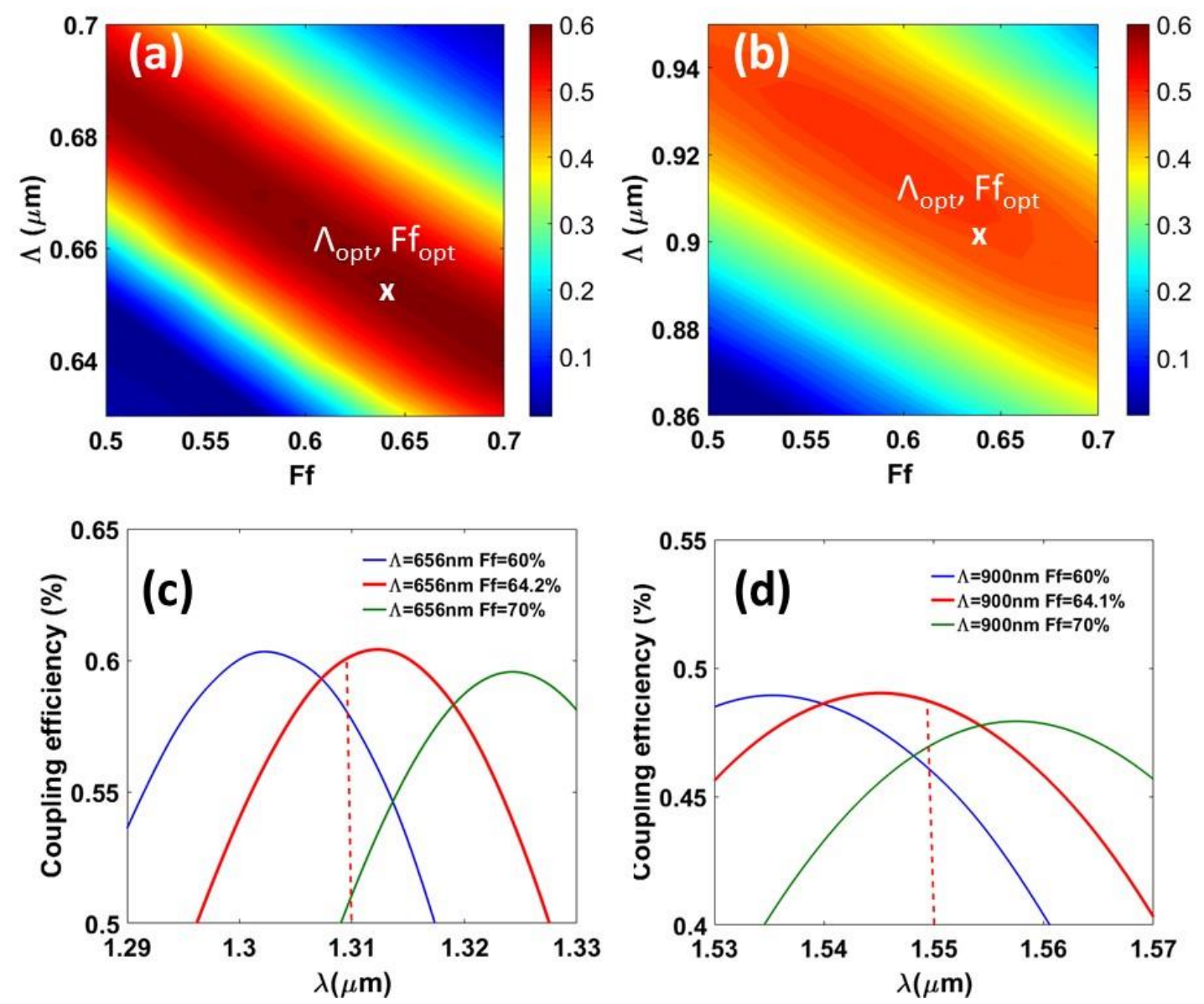

Figure. 7. Simulated coupling efficiency for TM polarization as function of the period and fill-factor for (a) $1310 \mathrm{~nm}$ and (b) $1550 \mathrm{~nm}$. The optimal values $\left(\Lambda_{\text {opt }}, F f_{\text {opt }}\right)$ obtained with the proposed design method are also shown. Transmission spectrum of the grating coupler for (c) $1310 \mathrm{~nm}$ and (d) $1550 \mathrm{~nm}$ for the optimum period and different fill-factor values.

Finally, the proposed method has also been used to design the optimum grating coupler for TM polarization. In this case, the new factor described by Eq. (11) is taking into account to obtain the effective index of the propagating mode in the grating. Therefore, the optimum values for the period and fill-factor are respectively $\Lambda_{o p t}=656 \mathrm{~nm}$ and $F f_{o p t}=64.2 \%$ for $\lambda=1310 \mathrm{~nm}$, which gives rise to a coupling efficiency of $60 \%$, and $\Lambda_{o p t}=900 \mathrm{~nm}$ and $F f_{o p t}=64.1 \%$ for $\lambda=1550 \mathrm{~nm}$, which yields to a coupling efficiency of 50\%. Figure 7(a) and Fig. 7(b) show the simulated coupling efficiency as function of the period and fill-factor and the optimum values obtained with the proposed design method respectively for $1310 \mathrm{~nm}$ and $1550 \mathrm{~nm}$. On the other hand, Figure 7(c) and Fig. 7(d) show the transmission spectrum of the grating for the optimum period and different fill-factor values for $1310 \mathrm{~nm}$ and $1550 \mathrm{~nm}$, respectively. It is again confirmed that maximum coupling efficiency of the transmission spectrum is achieved at the considered wavelength by using the optimum period and fillfactor.

\section{Conclusions}

A method has been proposed for designing high performance grating couplers by means of a semianalytic approach that avoids the optimization via extensive FDTD simulations. The key point is to calculate the optimum effective index of the propagating mode in the grating coupler by using a wavelength shifted from the target wavelength of operation. Thus, the optimum period and fill-factor are easily obtained with closed-form expressions that have been also formulated. The proposed method has been used for designing SOI grating couplers for both TE and TM polarizations with a high coupling 
efficiency above 50\% and the transmission spectrum centered around $1.31 \mu \mathrm{m}$ and $1.55 \mu \mathrm{m}$. Furthermore, it should be noticed that the proposed approach could also be applied to design uniform grating couplers in other waveguide geometries, such as rib or slot waveguides, or materials platforms by following the same methodology described here as the basis lies on the fundamental behavior of the grating coupler structure.

\section{Acknowledgment}

Funding from project TEC2016-76849 (MINECO/FEDER, UE) is acknowledged. Roberto Larrea also acknowledges the Ecuadorian Government for funding his grant.

\section{References}

[1] Hochberg, M., Baehr-Jones, T.: Towards fabless silicon photonics. Nat. Photonics 4, 492-494 (2010).

[2] Vivien, L., Pavesi, L.: Handbook of silicon photonics, Boca Raton: Taylor \& Francis (2013).

[3] Mekis, A., Gloeckner, S., Masini, G., Narasimha, A., Pinguet, T., Sahni, S., De Dobbelaere, P.: A Grating-Coupler-Enabled CMOS Photonics Platform. IEEE Journal of Selected Topics in Quantum Electronics, 17, 597-608 (2011).

[4] Vivien, L., Pascal, D., Lardenois, S., Marris-Morini, D., Cassan, E., Grillot, F., Laval, S., Fedeli, JM., El Melhaoui, L.: Light injection in SOI microwaveguides using high-efficiency grating couplers. Journal of Lightwave Technology, 24, 3810-3815 (2006).

[5] Van Laere, F., Roelkens, G., Ayre, M., Schrauwen, J., Taillaert, D., Van Thourhout, D., Krauss, T. F., Baets, R.: Compact and highly efficient grating couplers between optical fiber and nanophotonic waveguides. Journal Lightwave Technol. 25, 151-156 (2007).

[6] Alonso-Ramos, C., Ortega-Moñux, A., Molina-Fernández, I., Cheben, P., Zavargo-Peche, L., Halir, R., Janz, S., Xu, D.-X., Kim, N., Lamontagne, B.: Grating couplers for thick SOI rib waveguides. Optical and Quantum Electronics, 44, 535-540 (2012).

[7] He, L., He, Y., Pomerene, A., Hill, C., Ocheltree, S., Baehr-Jones, T., Hochberg, M.:Ultrathin Silicon-on-Insulator Grating Couplers. IEEE Photonics Technology Letters, 24, 2247-2249 (2012)

[8] Su, L., Trivedi, R., Sapra, N., Piggott, A., Vercruysse, D., Vučković, J.: Fully-automated optimization of grating couplers. Optics Express, 26, 4023(2018).

[9] Marchetti, R., Lacava, C., Khokhar, A., Chen, X., Cristiani, I., Richardson, D., Reed, G., Petropoulos, P., Minzioni, P.: High-efficiency grating-couplers: demonstration of a new design strategy. Scientific Reports, 7 (2017).

[10] Piggott, A., Lu, J., Babinec, T., Lagoudakis, K., Petykiewicz, J., Vučković, J.: Inverse design and implementation of a wavelength demultiplexing grating coupler. Scientific Reports, 4 (2014).

[11] Elachi, C.: Waves in active and passive periodic structures: A review. Proceedings of the IEEE, 64, 1666-1698 (1976).

[12] Bonod, N., Neauport, J.: Diffraction gratings: from principles to applications in high-intensity lasers. Advances in Optics and Photonics, 8, 156 (2016).

[13] Harris, J., Winn, R., Dalgoutte, D.: Theory and Design of Periodic Couplers. Applied Optics, 11, 2234 (1972).

[14] Halir, R., Bock, P., Cheben, P., Ortega-Moñux, A., Alonso-Ramos, C., Schmid, J., Lapointe, J., Xu, D., Wangüemert-Pérez, J., Molina-Fernández, I., Janz, S.: Waveguide sub-wavelength structures: a review of principles and applications. Laser and Photonics Reviews, 9, 25-49 (2014). 